\title{
Erratum to: Transarterial chemoembolisation (TACE) combined with endovascular implantation of an iodine-125 seed strand for the treatment of hepatocellular carcinoma with portal vein tumour thrombosis versus TACE alone: a two-arm, randomised clinical trial
}

\author{
MinJie Yang $\cdot$ ZhuTing Fang $\cdot$ ZhiPing Yan $\cdot$ \\ JianJun Luo $\cdot$ LingXiao Liu $\cdot$ Wen Zhang $\cdot$ LinLin Wu \\ JingQin Ma $\cdot$ QingHui Yang $\cdot$ QingXin Liu
}

Published online: 26 February 2014

(c) Springer-Verlag Berlin Heidelberg 2014

Erratum to: J Cancer Res Clin Oncol (2014) 140:

211-219

DOI 10.1007/s00432-013-1568-0

Few values in Tables 1 and 3 are inadvertently published wrongly in the original publication of this article. The corrected values with revised tables are listed below.
The online version of the original article can be found under doi:10.1007/s00432-013-1568-0.

M. Yang $\cdot$ Z. Fang $\cdot$ Z. Yan $\cdot$ J. Luo $\cdot$ L. Liu $\cdot$ W. Zhang $\cdot$ L. Wu J. Ma $\cdot$ Q. Yang $\cdot$ Q. Liu $(\varangle)$

Department of Interventional Radiology, Zhongshan Hospital, Fudan University, 180 Fenglin Road, Shanghai 200032, China e-mail: liu.qingxin@zs-hospital.sh.cn

\section{Z. Fang}

Department of Interventional Radiology, Provincial Hospital of Fujian Province, Teaching Hospital of Fujian Medical University, Fuzhou, China
Table 1 Baseline characteristics of patients

\begin{tabular}{|c|c|c|c|}
\hline Patient characteristics & $\begin{array}{l}\text { Group A } \\
(N=43)\end{array}$ & $\begin{array}{l}\text { Group B } \\
(N=42)\end{array}$ & $P$ value \\
\hline \multicolumn{4}{|l|}{ Demography } \\
\hline Age & & & 0.754 \\
\hline$>50$ year & 25 & 23 & \\
\hline$<50$ year & 18 & 19 & \\
\hline Sex & & & $1.000^{\mathrm{a}}$ \\
\hline Male/female & $39 / 4$ & $39 / 3$ & \\
\hline Aetiology & & & $0.298^{\mathrm{b}}$ \\
\hline HBV/HCV/alcohol & $40 / 2 / 1$ & $40 / 1 / 1$ & \\
\hline Morphology & & & 0.733 \\
\hline Multi-nodular/diffuse/massive & 15/19/9 & $18 / 17 / 7$ & \\
\hline Maximum diameter & & & 0.700 \\
\hline$>5 \mathrm{~cm}$ & 28 & 29 & \\
\hline$\leq 5 \mathrm{~cm}$ & 15 & 13 & \\
\hline$\alpha$-Fetoprotein & & & 0.593 \\
\hline$>400 \mathrm{ng} / \mathrm{mL}$ & 25 & 29 & \\
\hline$\leq 400 \mathrm{ng} / \mathrm{mL}$ & 18 & 13 & \\
\hline Location of tumour thrombosis & & & $0.778^{\mathrm{b}}$ \\
\hline $\mathrm{MPV}+\mathrm{RPVB}$ & 33 & 31 & \\
\hline $\mathrm{MPV}+\mathrm{LPVB}$ & 7 & 9 & \\
\hline MPV + RPVB + LPVB & 3 & 2 & \\
\hline Ascites & & & 0.922 \\
\hline Yes & 24 & 23 & \\
\hline No & 19 & 19 & \\
\hline Child-Pugh class & & & 0.756 \\
\hline A & 17 & 18 & \\
\hline B & 26 & 24 & \\
\hline ECOG & & & 0.379 \\
\hline $0 / 1 / 2$ & $8 / 26 / 9$ & $7 / 28 / 7$ & \\
\hline
\end{tabular}

$M P V$ main portal vein, $R P V B$ right portal vein branches, $L P V B$ left portal vein branches, massive tumour: maximum diameter $>10 \mathrm{~cm}$

a Fisher's exact test was used

b The likelihood ratio test was used 
Table 3 Significant factors associated with survival time in univariate analysis

\begin{tabular}{lclll}
\hline Characteristics & No. of cases & $\begin{array}{l}\text { Survival time } \\
\text { [Mean (SD) }\end{array}$ & $\chi^{2}$ & $P$ value \\
\hline Child-Pugh & & & 8.63 & 0.005 \\
A & 17 & $214.18(98.95)$ & & \\
B & 26 & $167.65(65.55)$ & & \\
ECOG & & & 15.59 & 0.000 \\
0 & 8 & $292.12(53.55)$ & & \\
1 & 26 & $197.27(77.04)$ & & \\
2 & 9 & $110.33(37.37)$ & & \\
PVTT & 9 & & 6.93 & 0.012 \\
Response & 34 & $260.44(108.82)$ & & \\
Non-response & 34 & $179.55(73.58)$ & & \\
Intrahepatic response & & 25.32 & 0.000 \\
CR + PR & 10 & $293.50(102.40)$ & & \\
SD + PD & 33 & $167.39(56.79)$ & & \\
\hline
\end{tabular}

